\title{
La empleabilidad, sus sentidos y dispositivos: un estudio de caso desde la perspectiva de los beneficiarios del Programa Jóvenes Más y Mejor Trabajo en Córdoba Capital, Argentina
}

\author{
Gonzalo Assusa \\ Doctorando en Ciencias Antropológicas (Universidad Nacional de Córdoba) \\ Investigador de la Universidad Nacional de Córdoba \\ Córdoba, Argentina \\ gon_assusa@hotmail.com \\ María Gabriela Brandán Zehnder \\ Doctoranda en Ciencia Política (Universidad Nacional de Córdoba) \\ Investigadora y docente de la Universidad Católica de Córdoba \\ Córdoba, Argentina \\ mgbrandanzehnder@gmail.com
}

\begin{abstract}
Resumen
En la última década asistimos a una reconfiguración de las políticas de empleo y del lugar del Estado como regulador de las prácticas laborales. Bajo el paradigma de las políticas activas de empleo, la intervención, en particular sobre la problemática de empleo juvenil, se ha dado a partir de la categoría de empleabilidad, poniendo el foco en procesos de orientación e inducción al mundo del trabajo. Desde una perspectiva que piensa las políticas públicas de empleo como dispositivos de subjetivación, este texto recupera los análisis realizados sobre un programa de empleo juvenil de Argentina, para enfocarse en la mirada de los beneficiarios y analizar los desplazamientos y la producción de sentidos sobre el trabajo en el marco habilitado por el programa.
\end{abstract}

Palabras clave: empleo juvenil, políticas públicas de juventud, empleabilidad, dispositivos, modos de generación.

\section{Introducción}

Durante la última década asistimos a un viraje en los modos de gestión gubernamental del trabajo en Argentina que importó una modificación del perfil de las políticas orientadas a fomentar el empleo y la inserción laboral. ${ }^{1}$ Esta transformación se asoció a la definición

1. Tal como sostiene Madoery, "durante el período 2003-2010 las políticas han adquirido las siguientes características: incentivos sectoriales; políticas asistencialistas y mecanismos de ayuda económica a sectores vulnerables; actuación sobre públicos específicos, como los jóvenes, con programas de igualdad de oportunidades y de fomento a la empleabilidad y el emprendedorismo; formación profesional y capacitación en oficios; transferencia de responsabilidades y recursos a colectivos sociales determinados; adecuación de los marcos legales que regulan los mercados de trabajo; mejoras en las capacidades institucionales del propio ministerio (creación de oficinas de intermediación laboral), apertura de espacios de diálogo y concertación entre el gobierno y los sectores de la producción y el trabajo, con el propósito de mejorar las relaciones laborales y la calidad del empleo" (Madoery, 2011, p. 8). 
de políticas activas de empleo ${ }^{2}$ e implicó cambios en los modos de intervención con aristas diversas, pero referidas al menos a tres dimensiones: a) el pasaje del rol del Estado desde una modalidad más enfáticamente asistencial a una de promoción; así como también, b) desde un enfoque orientado a la contención frente al desempleo, a uno con miras a la mejora de la empleabilidad; y finalmente, c) una redefinición de los sujetos destinatarios, tanto respecto de los grupos poblacionales atendidos, como del abordaje dispuesto en clave de "derechos" de los mecanismos dirigidos a los mismos.

Uno de estos grupos especialmente tratados por los dispositivos gubernamentales son los jóvenes, quienes, pese a la recuperación en materia de empleo que ha vivido el país en los últimos 10 años, continúan con dificultades diferenciales a la hora de insertarse en el mundo del trabajo ${ }^{3}$ respecto de otros grupos de la PEA. Así, los condicionantes individuales de quienes se encuentran en esta franja etaria (falta de terminalidad educativa, ausencia de experiencia laboral, baja capacitación profesional, desinformación en cuanto a los mecanismos de búsqueda etc. (OIT, 2011, 2012, 2013), como aquellos propios del mercado de trabajo (segmentación, heterogeneidad, etc.) sumado a la temporalidad de sus situaciones de desempleo (origen en la inactividad, trayectorias laborales precarias e inestables, etc.), los convierten en poblaciones especialmente vulnerables, a los ojos de la mirada estatal.

De esta manera, desde el año 2008 se implementa en Argentina el Programa Jóvenes con Más y Mejor Trabajo (PJMyMT), dispositivo central del Ministerio de Trabajo, Empleo y Seguridad Social de la Nación (MTESS), diseñado para la mejora de la empleabilidad de jóvenes de 18 a 24 años de edad, sin terminalidad educativa. Dicho programa se ha convertido en el mecanismo gubernamental más relevante en el tratamiento de la problemática de empleo juvenil en los últimos años, no solo por la extensión territorial del mismo y/o la cantidad de beneficiarios a los que atiende, ${ }^{4}$ sino además por el enfoque propuesto en el tratamiento de la misma.

Partimos del supuesto de que estas prácticas de intervención gubernamental no solo se orientan a atacar la causalidad de la problemática vinculada al empleo, sino que además pretenden definir los contornos del campo de acción de los sujetos destinatarios. Esta idea se sustenta en una lectura de las políticas públicas como dispositivos de poder que, desde una mirada gubernamental, operan como estrategias para la reducción de las condiciones de inempleabilidad $\mathrm{y}$, en consecuencia, de ese conjunto de población desempleada, al tiempo que desarrolla técnicas específicas de gobierno que fijan los límites, lo enunciable y lo posible de ser inscripto en una u otra posición, una "biopolítica" montada para administrar lo que es el trabajo, qué condiciones se requieren para él y quiénes acceden al mismo.

Esta perspectiva, como veremos más adelante, se presenta como una mirada alternativa frente a los estudios dominantes en la sociología de la juventud, en tanto propone reparar en la dimensión institucional que participa del proceso de inserción, no como una mera instancia de mediación entre los sujetos y sus condicionantes, sino como un ámbito de producción de subjetividades, que responde a esquemas ideológicos dominantes en torno a la juventud y al trabajo.

Tal como lo entendemos, el PJMyMT y los diagnósticos que lo sustentan - ya sea a nivel ministerial como en el ámbito de diferentes organizaciones internacionales - suponen no sólo una matriz explicativa respecto del mundo del trabajo y sus problemáticas, sino también una matriz epistemológica que toma a la juventud como un grupo existente "de hecho", operando con cierta "homogeneidad", y pasible de ser intervenido. ${ }^{5}$

2. De acuerdo con la OIT, las políticas activas de empleo, que forman parte de las políticas activas del mercado de trabajo (PAMT), se refieren al conjunto de las normas y orientaciones de un Estado para promover la inserción de las personas - en edad de trabajar - en el mercado de trabajo. Comprenden las directrices mismas acerca de cómo debe realizarse tal promoción y la forma en que esta acción del Estado debe integrarse al resto de las políticas públicas. También implican medidas para favorecer la ejecución de programas que potencien la inserción o reinserción de los trabajadores en el empleo: servicios de empleo e intermediación laboral; formación profesional y desarrollo de las calificaciones; creación y promoción de puestos de trabajo en el marco de programas de obras públicas o comunitarias; fomento de la creación de empresas; fortalecimiento de la actividad productiva local; subsidios a la contratación, etc. (OIT, 2008). Las mismas se convierten en el paradigma que rige las acciones del MTEySS en el período posterior a la crisis social y económica del año 2001, y su puesta en práctica ha importado una continua cooperación técnica con la OIT.

3. De acuerdo a Salvia, "la tasa de desocupación de los jóvenes ubicados entre los 16 y los 24 años casi cuadriplica a la de la población que se sitúa entre los 25 y los 64 años. Esta marcada diferencia ha persistido en niveles elevados desde 2003 hasta la fecha. Cabe mencionar que aunque después de la crisis de 2001-2002 la tasa de desocupación de los jóvenes descendió, esta se ha mantenido en torno al 19 por ciento, mientras que la tasa de desempleo de los adultos declinó de manera importante (de 13 por ciento a 5,5 por ciento). [...] A esta tasa de desempleo habría que sumar el problema del subempleo de subsistencia, el cual afecta especialmente a los jóvenes de los sectores pobres. En total más del 20 por ciento de los jóvenes económicamente activos se han visto afectados por diferentes formas de desempleo 'oculto'" (Salvia, 2013, p. 4).

4. De acuerdo a un informe interno del MTEySS, en el año 2011 los beneficios que otorga el programa alcanzaron en Argentina a 300000 jóvenes. (Fuente: Informe de monitoreo mensual del MTEySS).

5. Tal como lo muestra Chaves (2010), la construcción del objeto (de investigación) no puede pensarse por separado respecto de su intervención: la juventud emerge, en América Latina, y se vuelve relevante como objeto en la agenda académica, en su visibilización como problema social en la agenda pública internacional. 
En primer lugar, los discursos que se imponen en un momento sobre los problemas sociales a tratar y las soluciones a los mismos no son meros reflejos de una realidad que les preexistiría. Por el contrario, para que exista un problema social es necesario que alguien lo delimite como tal: que trace las fronteras de una población o ámbito a gestionar -a tratar, a modificar [...]. En segundo lugar, discursos y categorías no son una mera cuestión de conocimiento, técnica. Por el contrario, en la medida en que logran imponerse como la representación legítima, verdadera del mundo social van a producir efectos reales sobre este mundo. (Martín Criado, 1999, p. 17)

Intentando salir del vicio sustancialista de captar una generación como algo dado y de adjudicarle una serie de características (problemática, perdida, sin valores, marcada por carencias, peligrosa, etc.), pretendemos reconstruir este singular programa de empleo como un contexto en el que se definen modos de generación (Martín Criado, 1998), es decir, formas de producción y modulación de subjetividades en torno a la "edad" (generaciones) y al "trabajo" (inempleables), ámbitos que, antes que transmitir los valores necesarios para la "correcta" inserción en el mundo del trabajo, generan procesos de valorización de los sujetos, de sus trayectorias y sus recursos (Martín Criado, 1999).

En esta oportunidad, pretendemos reparar en las apropiaciones que los jóvenes que participan del programa desarrollan respecto de los sentidos en torno al trabajo y al juego de negociaciones sobre la interpelación "generacional" que opera sobre ellos. Entendemos que las relaciones que se puedan dar entre ambas lógicas (aquella propia del Estado y sus agentes, y la de los sujetos) no aparecen de manera lineal sino que habilitan un haz de posibilidades y entrecruzamientos para definir el problema político-cultural que tensiona la cuestión de la "juventud" y la "empleabilidad".

De esta manera, a partir del análisis de entrevistas realizadas a beneficiarios del programa a lo largo de los años 2011, 2012 y 2013 en la ciudad de Córdoba (Argentina), identificaremos las trayectorias laborales de los jóvenes y sus sentidos asociados al trabajo, reconoceremos los formatos de incorporación de las prácticas del dispositivo en sus trayectorias laborales y analizaremos la presencia y disposición de valores propios de lo que llamamos "cultura de la empleabilidad" en los discursos de los beneficiarios.

\section{Breve presentación del caso: el Programa Jóvenes con Más y Mejor Trabajo}

El PJMyMT nace en el año 2008 en el marco general del Plan Integral para la Promoción del Empleo "Más y Mejor Trabajo", a cargo del MTESS. Este dispositivo está orientado a jóvenes de entre 18 y 24 años de edad que posean estudios secundarios incompletos y supone el diseño y desarrollo de un conjunto de instituciones estatales con miras a la mejora de las condiciones de empleabilidad y a la generación de oportunidades de inclusión social y laboral de los jóvenes. ${ }^{6}$

Su gestión se apoya en las capacidades institucionales en el nivel local -particularmente de los municipios- por medio de los servicios de empleo. Incluye una asignación económica por beneficiario por un plazo que varía entre los 2 y los 18 meses condicionados al cumplimiento de compromisos específicos vinculados a la participación en actividades del programa.

Pese a que estas prestaciones se presentan de manera general, el acceso a una u otra estará mediada por la primera instancia del programa: la orientación e inducción al mundo del trabajo. Aquí se desprende un elemento que define la particularidad de este dispositivo en relación a otro tipo de intervenciones estatales orientadas a la inclusión laboral juvenil: ${ }^{7}$ el joven accederá a las diferentes prestaciones de acuerdo al proyecto formativo ocupacional decidido y definido por él mismo.

El Proyecto Formativo Ocupacional es un constructo en base a la historia personal de cada uno de los beneficiarios que da cuenta de sus trayectorias educativas y formativas así como también de sus experiencias laborales previas. Este proyecto es individual y resume las herramientas con las cuales el joven cuenta para acceder al mercado de trabajo y aquellas de las que carece, y que debe obtener en el marco del programa. Si bien las prestaciones son generales y se presentan como acciones integradas, los recorridos que cada uno de los jóvenes emprende son particulares, generando, en términos del programa, diferentes "circuitos operativos" (Res. 461/2008). desempeñarse, finalizar su escolaridad obligatoria, realizar experiencias de formación y/o de prácticas calificantes en ambientes de trabajo, iniciar una actividad productiva de manera independiente o insertarse en un empleo" (Art. 1, Res. 497/2008).

7. Como bien reseña Rodríguez (2011), las respuestas estatales ante la problemática juvenil con el trabajo en la región latinoamericana han ido variando a lo largo del tiempo, en relación a los diagnósticos que se presentaban. Así, desde mediados de los 90 surge una serie de políticas orientadas a lograr la inclusión laboral de este sector poblacional, que se desplaza en un arco que va desde programas destinados a fortalecer las competencias educativas (formales y profesionales), garantizar el primer empleo, fomentar el emprendedorismo, orientar e informar a los jóvenes en la búsqueda de trabajo, etc. 
Juventud e inserción laboral: algunos elementos conceptuales para pensar la relación entre políticas públicas, ingreso al mercado de trabajo y procesos de subjetivación

\section{La sociología de la juventud y el enfoque de las transiciones: la dimensión institucional de la inserción como mediadora}

La mirada sobre la juventud desde la perspectiva de las transiciones atiende a las múltiples trayectorias e itinerarios que los jóvenes siguen en su paso a la vida adulta. Como sostienen Casal et al. (2006), se entiende por transición el conjunto de procesos biográficos de socialización que de forma articulada intervienen en la vida de las personas desde que asumen la pubertad y que proyectan al sujeto joven hacia la consecución de la emancipación profesional y familiar y a la adquisición de posiciones sociales. Es decir, es un proceso de adquisición con respecto a las relaciones de producción y reproducción.

Dos grandes transiciones entonces, caracterizarían a la juventud: una vinculada a la esfera productiva (transición escuela-trabajo) y otra a la familiar (dependencia familiar-independencia). En este sentido, la sociología de la juventud se refiere a las primeras como al "conjunto de adquisiciones, expectativas y acciones del actor estudiante y su concreción en un proceso de posicionamiento social o enclasamiento en la estructura ocupacional" (Casal et al., 2006, p. 20).

Es relevante señalar que los modos que adoptan esas transiciones han sufrido importantes cambios; tal es así que la "cuestión juvenil" (Salvia, 2008) ha puesto en evidencia la heterogeneidad de las transiciones juveniles conforme las circunstancias socioeconómicas, políticas y culturales en las que los diferentes sujetos se encuentran. Esto implica atender, como sostienen Dávila, Ghiardo y Medrano (2006), tanto los factores o variables de orden estructural en los que se insertan estas transiciones como el carácter exitoso o fallido de un determinado proceso de transición.

Sin embargo, hace algunos años algunos estudiosos pertenecientes a esta tradición han señalado la importancia de las dimensiones subjetivas en el análisis referidas a las aspiraciones y expectativas de los jóvenes, $y$, en este sentido, los abordajes han hecho énfasis en el análisis de períodos más largos y en las trayectorias laborales individuales, como elementos que permitirían comprender con más profundidad la complejidad del proceso de inserción laboral (Jacinto, 2010).

Esta mirada no rechaza un abordaje que ponga en cuestión los aspectos estructurales que conforman la matriz objetiva en la que se encuentran insertos los sujetos, sino que por el contrario pretende dar cuenta de la capacidad de agencia de los individuos en sus transiciones al mundo del trabajo, a partir de considerar el capital social y cultural que disponen a lo largo de las mismas. Como sostiene Jacinto, "las transiciones reflejan, al mismo tiempo, voluntades personales y condicionantes estructurales y contextuales, que se conjugan dinámicamente y diversifican los recorridos laborales" (Jacinto, 2010, p. 25).

De esta manera, las trayectorias e itinerarios de los jóvenes estarían atravesados por ambas dimensiones. Empero, la proliferación reciente de numerosos dispositivos estatales destinados a los jóvenes es un indicador de la relevancia que adquiere la dimensión institucional en el análisis de la inclusión laboral juvenil, en un contexto signado por déficits en las tasas de ocupación juvenil y la necesidad de repensar estrategias de inclusión acordes a los desafíos actuales.

Así, de acuerdo a esta perspectiva, las instituciones se constituyen en instancias mediadoras entre las condiciones estructurales del proceso de inserción y las condiciones subjetivas. ${ }^{8}$ De allí que el abordaje del éxito o fracaso de las políticas y programas que se efectúa desde esta mirada se orienta a evaluar la incidencia de los mismos en las trayectorias de los jóvenes o al análisis de las estrategias y/o capitales que los mismos ponen en juego en el marco de estos dispositivos (Jacinto y Millenaar, 2013; Burgos y Roberti, 2013).

Pese a que esta lectura ha realizado un aporte significativo al campo de la interpretación sobre las transiciones juveniles al mundo del trabajo, pretendemos dar cuenta a continuación de que es posible invertir la mirada sobre los dispositivos gubernamentales, no a partir de un análisis de sus resultados sino del modo mismo en que estos operan, entendiendo que en los mecanismos que desarrollan se despliegan prácticas tendientes a producir "jóvenes empleables" que responden a un conjunto de construcciones ideológicas.

\section{Una lectura desde la gubernamentalidad: las instituciones como productoras de sujetos}

En este sentido, consideramos que las instituciones no son meras instancias mediadoras entre las condiciones materiales-objetivas problemáticas de los jóvenes y sus construcciones subjetivas; sino que, por

8. El término institución aquí se toma en un sentido amplio. Como menciona Jacinto, "nos referimos a las instituciones que intervienen en el proceso de transición desde las escuelas hasta los servicios de empleo pasando por los centros de formación profesional y organizaciones sociales" (Jacinto, 2010, 29). 
el contrario, el diseño institucional se encuentra profundamente imbricado en la forma en que los sujetos se reconocen a sí mismos. La dimensión institucional aparece, de esta manera, como instancia productora de subjetividad, entendiendo por tal no un estado sino un proceso, ${ }^{9}$ que no depende exclusivamente de la agencia del sujeto a partir de las circunstancias sino que se encuentra profundamente entrelazada en las mismas.

En otras palabras, quisiéramos proponer una lectura de los dispositivos orientada a dar cuenta de la operatoria de la institución a partir de los mismos, como parte de un entramado vasto de relaciones de poder en torno a la juventud y el trabajo, a fin de comprender de qué manera esas transiciones se insertan en procesos político-hegemónicos más amplios, ${ }^{10}$ es decir, en un conjunto de gubernamentalidades que luego pueden resultar estatizadas. ${ }^{11}$

Esta lectura, no obstante, no pretende olvidar que en el análisis de las prácticas que habilita el dispositivo existen una serie de brechas, puntos de fuga y márgenes de posibilidades, en el marco de los cuales, el campo de acción que éstos intentan, a la vez, producir y regular, se desenvuelve y constituye configuraciones diversas, con sentidos disímiles.

Tal como las entendemos, las políticas públicas funcionan como instanciaciones de racionalidades de gobierno, que regulan los hábitos, rutinas y procedimientos de los sujetos (Roseberry, 2002, p. 216), como así también influyen sobre las categorías de individuo y subjetividad, es decir, sobre las lógicas cla- sificatorias de los agentes (Shore, 2010, p. 37). ${ }^{12}$ Sin embargo, también es importante remarcar que estas lógicas fijadas - muchas veces eficazmente - por las políticas públicas, toman por momentos derivas inesperadas en las prácticas y significaciones de los agentes. ${ }^{14}$ Así, si bien las políticas de empleo habilitan una serie de sentidos en torno al trabajo, por momentos, éstos son subvertidos, desplazados y/o modulados por sus destinatarios, produciendo un efecto desestabilizador sobre las bases de legitimación de esta política.

\section{Disputando sentidos en torno al trabajo: la mirada de los jóvenes}

Uno de los supuestos fundamentales del PJMYMT es que los jóvenes tienen grandes dificultades para insertarse en el mercado de trabajo, a partir de su deficiente empleabilidad. Esto, como sostiene Salvia, se sustenta en un "diagnóstico que explica los desajustes en las competencias educativas y laborales, ubicando a la 'oferta' como factor explicativo del mayor desempleo juvenil, sobre todo en sectores de poca o nula calificación laboral" (Salvia, 2013, p. 8). No obstante, como hemos analizado previamente, la empleabilidad ${ }^{15}$ desde el discurso de los funcionarios se desplaza desde un registro "formativo-educativo" con eje en competencias para el trabajo, hacia un aspecto de índole "motivacional-actitudinal", esto es,

9. Señala Tassin (2012): "la subjetivación designa un proceso y no un estado (una situación, un estatus o un principio del ser). Pero este proceso no es simplemente el de un llegar a ser sujeto, como si pudiera darse por entendido que sabemos lo que significa 'ser sujeto': es más bien el proceso de un llegar a ser ' $x$ ', proceso que no sabría fijarse, estabilizarse bajo la forma de 'sujeto', sea cual sea el sentido en el que se tome el término, bien sea en el sentido de la subjetidad, de la subjetividad o de la sujeción."

10. No queremos con esto decir que la Sociología de la Juventud, desde el enfoque de las transiciones no haya reparado en esto ya que, por ejemplo, Bendit y Stokes (2004) sostienen que existe desde una construcción político-administrativa de la desventaja social como base para la legitimación de las políticas dirigidas al sector. No obstante, nuestra propuesta es ir un paso más allá y poder insertar esta estrategia de legitimación en el marco de una economía de relaciones de poder orientadas al mundo del trabajo en general y a los jóvenes en particular. Tal como sostiene Cris Shore, "las políticas reflejan maneras de pensar el mundo y cómo actuar en él. Contienen modelos implícitos -y algunas veces explícitos- de una sociedad y de visiones de cómo los individuos deben relacionarse con la sociedad y los unos con los otros" (Shore, 2010, p. 31).

11. Por prácticas de gubernamentalidad se entiende el poder visto como un ámbito de relaciones que suponen, en sentido amplio, conducir la conducta de los hombres.

12. Sostiene Foucault: "Esta forma de poder emerge en nuestra vida cotidiana, categoriza al individuo, lo marca por su propia individualidad, lo une a su propia identidad, le impone una ley de verdad que él tiene que reconocer y al mismo tiempo otros deben reconocer en él" (Foucault, $1983, \mathrm{~s} / \mathrm{d})$

13. Esta mirada sobre el poder sólo es posible en tanto los sujetos son libres. Señala Foucault: “Cuando se define el ejercicio del poder como un modo de acción sobre las acciones de los otros, cuando se caracteriza esas acciones como el gobierno de los hombres por otros hombres, -en el sentido más amplio del término- se incluye un elemento muy importante: la libertad. El poder sólo se ejerce sobre sujetos libres, y sólo en tanto ellos sean libres. Por esto entendemos sujetos individuales o colectivos que están enfrentados a un campo de posibilidades en el cual diversas formas de comportarse, diversas reacciones y comportamientos pueden ser realizados" (Foucault, 1983, s/d).

14. Esta mirada sobre el poder sólo es posible en tanto los sujetos son libres. Señala Foucault: "Cuando se define el ejercicio del poder como un modo de acción sobre las acciones de los otros, cuando se caracteriza esas acciones como el gobierno de los hombres por otros hombres, -en el sentido más amplio del término- se incluye un elemento muy importante: la libertad. El poder sólo se ejerce sobre sujetos libres, y sólo en tanto ellos sean libres. Por esto entendemos sujetos individuales o colectivos que están enfrentados a un campo de posibilidades en el cual diversas formas de comportarse, diversas reacciones y comportamientos pueden ser realizados" (Foucault, 1983, s/d).

15. La noción de empleabilidad no es nueva en el contenido de los programas de inclusión laboral juvenil, aunque ha adquirido diferentes sentidos a lo largo de los años. Para una lectura más amplia sobre esto, véanse Pérez (2005) y Jacinto (2008). 
como una carencia por parte de los jóvenes a la hora de conseguir empleo debido a una serie de limitaciones propias de: a) su grupo generacional; b) las condiciones puntuales de los jóvenes que acceden a este dispositivo (jóvenes de "sectores vulnerables").

Este diagnóstico, que da sustento a una mirada activa sobre la labor del Estado - tanto en su dimensión reguladora y promotora como educativa y formadora -, puede desagregarse en las siguientes dimensiones:

1) Restricciones formativas para el trabajo, tanto en lo que refiere a competencias educativas básicas como profesionales; 2) Carencia de hábitos o disposiciones para el trabajo, ya sea producto de inexperiencia o de herencias familiares de desocupación y asistencia crónica; 3) Falta de interés y motivación en un proyecto de vida futuro, característica propia de la juventud, y más fuertemente, de este sector de jóvenes con condiciones de vulnerabilidad social (ya que provienen de contextos conflictivos vinculados, de acuerdo a los funcionarios, a las drogas, la violencia y el delito).

La intención de este apartado es construir una mirada invertida de este diagnóstico - atendiendo principalmente a las últimas dos dimensiones - a partir de los discursos de los jóvenes que participan del programa, a fin de poner en tensión los supuestos del dispositivo y dar cuenta de los sentidos juveniles sobre el trabajo.

\section{Trayectorias y sentidos: experiencias, méritos y realización personal}

Uno de los equívocos de los que el PJMyMT parte, y que condiciona todo su diagnóstico acerca de la problemática de la "empleabilidad" de los jóvenes, es el de la "falta de experiencia laboral" de esta población. Una de las referencias más recurrentes en el discurso de los agentes del equipo técnico del programa, como así también en el de funcionarios, es la caracterización de estos jóvenes como una posible "generación perdida": hijos formados en hogares donde persiste el desempleo y la "cultura de la asistencia” por, al menos, dos generaciones. ${ }^{16}$

En el relato de los jóvenes entrevistados, sin embargo, su trayecto ingresa al mercado laboral a temprana edad, ${ }^{17} \mathrm{y}$ este ingreso es justificado de diversas maneras: la necesidad de un ingreso económico extra para la unidad familiar, la búsqueda de alternativas para ocupar su tiempo libre tras situaciones de deser- ción escolar, por un interés personal en la búsqueda de independencia económica para los gastos propios, como experiencias de aprendizaje etc.:

Trabajé en la granja del zoológico, [...] era gracioso, divertido. En realidad me anoté con una amiga porque, más que todo como una experiencia como era. Si bien a mí no me hace falta, o sea, económicamente estoy bien [...]. Y yo quise hacerlo sola, y por eso fui a aprender, así, diferentes trabajos, diferentes cosas y era, más que todo era una prueba a mí, a ver si tenía la responsabilidad suficiente y esas cosas. Para tener más confianza. Y bueno, eso hice y por eso trabaje en esos diferentes trabajos

Yo empecé... hice primer año y me fue para la mierda (sonríe en la pausa), me llevé hasta el banco, me llevé... así que... Dejé. Dejé y a mi viejo no le gustó, y bueno, si dejás el colegio, bueno, vaya a trabajar, dice... “Acá no va a estar al pedo". Y bueno, primero empecé a trabajar en las obras y...

A diferencia de lo sostenido por el discurso del ministerio y de los organismos, los jóvenes no se muestran como aquellos "inactivos" totalmente desanclados del mundo del trabajo. Por otra parte, sus familias no constituyen tampoco ámbitos signados por el desempleo. Aun cuando muchos manifiestan conocer y tener contacto con una diversidad de políticas sociales y de asistencia a través de sus familias, el trabajo es una constante en la vida de todos sus padres: docentes, albañiles, pintores de obra, panaderos etc. La gran mayoría realizando tareas no calificadas o de calificación operativa. La familia, en este sentido, aparece como factor fundacional y determinante de sus propias trayectorias laborales en, al menos, dos dimensiones.

Por un lado, aparece como fuente fundamental para desplegar búsquedas laborales. Éstas se encuentran estrechamente ligadas a redes familiares, de vecindad o amistad. Los vínculos laborales que lograron establecer se realizaron generalmente por intermedio de conocidos que mediaron con demandantes de fuerza de trabajo. No aparecen en sus relatos instancias de entrevistas previas de empleo y/o de entrega de curriculum vitae (CV) como mecanismos, sino relaciones tramadas en términos de proximidad y vínculos personalizados. Esta modalidad aparece como una estrategia alternativa a la forma hegemónica propugnada por el discurso de los recursos humanos, desplegada por los jóvenes que pertenecen a sectores [producidos como] vulnerables, ante una lógica de selección que los excluye de antemano:

16. Para ver un análisis más detallado de las implicancias de este discurso, véase Assusa y Brandán Zehnder (2014).

17. Las edades aproximadas de ingreso al mercado laboral, para el caso de los jóvenes aquí entrevistados, rondan entre los 13 y los 15 años para los varones, y entre los 15 y los 17 años en el caso de las mujeres. 
No está nada fácil conseguir un trabajo [lo dice apesadumbrado, en voz baja]. Si no tenés el secundario hecho no podés conseguirlo. No, está complicado. Por otra parte, si vivís en una villa se te complica mucho más todavía. Por qué, porque "¿vos vivís en una villa?", “Sí, yo vivo en La Lonja, ponele”. Es como, "bueno, después te llamamos", y no te llaman... por qué, porque vivís en una villa y lo primero que se le pasa por la cabeza es que este es un drogadicto, un chorro, un narcotraficante, y vos ¿Qué decis? Te da por las bolas, ¿entendés? Que la gente esté pensando eso de vos, y sin conocerte bien, ¿entendés? Aparte, vos me conocés y sabés muy bien que yo no robo, o sea... mucha gente me conoce y sabe bien lo que soy yo, cómo me porto en la calle, cómo afronto la vida, qué hago para poder comer y sobrevivir ¿entendés? Pero hay gente que no te conoce y vos vas a una entrevista, estás perdido, ¿entendés?

A partir de esto, los tipos y ramas de actividades en las que los jóvenes se desempeñan o desempeñaron presentan muchas continuidades con sus trayectorias laborales familiares. Así, la condición de informalidad, más que la de desempleo, se plantea como un aspecto común entre las historias laborales de los entrevistados y la de sus padres. Casi ninguno de los padres de los entrevistados contaba con trabajos registrados y estables.

Sobre esto, debemos señalar varias líneas de explicación. Es posible que el carácter informal y no registrado de las experiencias laborales de los entrevistados esté vinculado en alguna medida al ingreso precoz de muchos de estos jóvenes al mercado de trabajo. Pero además, al ámbito familiar o personal en el que desenvuelven sus actividades laborales, donde la relación construida se define más a partir de la lógica del don (favor de otorgar el puesto de trabajo y brindar esa primera oportunidad), que por la lógica del derecho y la consiguiente obligación de registrar formalmente esta tarea, funcionan, a la vez, como un condicionante y una legitimación para su condición de precariedad. En este sentido, algunos de los jóvenes entrevistados no perciben como injusta la situación precaria de su empleo. Muchos de ellos "aceptan" estas condiciones y consideran que "no hay que hacer lío para no irse mal y poder volver". La noción de trabajos registrados y estables aparece proyectada en el marco de futuras etapas de su vida (cuando tengan su casa, hijos etc.). El atravesamiento de instancias formativas y de reflexión del PJMyMT en torno al derecho laboral y a sus propias experiencias, aparece en sus relatos como un quiebre o un momento de conciencia sobre sus vivencias laborales.

Por otra parte, el segmento del mercado laboral donde estos jóvenes se insertan (construcción, pequeños talleres de mecánica automotriz, servicio doméstico, ventas, etc.) se caracterizan por ser ramas de actividad mucho más expuestas a los fenómenos de informalidad y precariedad. ${ }^{18}$

En segundo lugar, la familia aparece por momentos como una fuente de saberes para el trabajo. En el caso de los varones entrevistados, muchos rescatan el aprendizaje de un oficio en el seno familiar, que luego les permitió el ingreso al mercado de trabajo.

Después empecé trabajando ayudándolo a mi viejo en la pintura... pintura de obra. Y hacer changuitas, ${ }^{19} \mathrm{y}$ después ya fui aprendiendo el oficio y ya empecé a hacer trabajos, pinturas, en la casa de mi tía, y así... es más, el otro día le pinté la casa a un chico amigo... tenía que entregar la casa... se iba a otro lado, y le he pintado todo adentro.

Claro, digamos, aprendí con mi viejo, acá en mi casa. Afuera no fui a ningún lado. Aprendí con mi viejo, viste, a... me enseñó a revocar, a levantar las paredes... cómo se debe trabajar, digamos.

En el caso de las mujeres entrevistadas, su desempeño se vincula a las competencias asociadas a la posición ocupada hacia el interior del hogar: el trabajo vinculado al espacio doméstico, al cuidado de personas y al trato con personas (en el comercio). Ambas dimensiones implican una ruptura con la construcción discursiva de los agentes del programa acerca de las familias de los jóvenes vulnerables como causal fundamental del problema: un espacio de formación personal del joven en un ámbito de ausencia de trabajo, con trayectorias vinculadas a la asistencia social y a la pérdida del trabajo como "valor".

Aun cuando sus trayectorias rompan con algunos de los supuestos del diagnóstico ministerial, muchos de los sentidos producidos por los jóvenes en torno al trabajo coinciden con las líneas habilitadas por el programa en cuanto a formación de disposiciones subjetivas. Así, encontramos que las significaciones fundamentales en torno a la motivación para su trabajo se vincula a las nociones de autonomía individual y de activación (económica), propias del imaginario de las políticas activas: sus actividades laborales se fundan en buscar independencia económica en sus hogares, para sus gastos, y en la búsqueda de realización a nivel personal, de sentirse capaces y con "poder" para "hacer".

18. De acuerdo a los datos del MTESS para el año 2011 en todo el país, junto con la rama del "comercio", la de "construcción" y la del "servicio doméstico" (con un $13 \%$ y un $23 \%$ del total de empleados no registrados, respectivamente) son las más expuestas al fenómeno de empleo no registrado. Lo mismo sucede con las ocupaciones no calificadas, que representan un $50 \%$ del total de empleados no registrados del país.

19. En el lenguaje coloquial, trabajos temporales, cortos y no registrados. 
Mayormente depende de las metas, a mí me gustaría trabajar porque, me gustaría trabajar pero mayormente para tener mis cosas y todo eso. No tener que estar: "Me das plata para esto". Con tus propias cosas, con tu propia plata podes comprarte lo que vos querés, independizarte y todo eso.

No, es sentir orgullo así de que uno, de que uno puede ser lo, de hacer por tu propia voluntad, claro y que uno solo puede $[. .$.$] trabajar es sentir que uno puede, entonces$ es como que te da más, no sé, para mí me daba más autoconfianza, me daba más...para mí es importante digamos. Básicamente es eso para mí.

Por otra parte, más allá de la efectiva continuidad de sus actividades laborales y las de sus padres, al proyectar sus deseos en el mundo laboral encontramos un fuerte desplazamiento respecto de las trayectorias familiares.

El trabajo que prefiero yo es el trabajo de estar sentado y estar en la oficina y estar ahí nomás.

Y, yo siempre quise entrar a una fábrica... de Fiat... [...]. A alguna fábrica de motores, de autos. [...] Siempre quise entrar ahí. Pero si no tengo esa posibilidad... abrirme un taller así como ha hecho José... pero no básicamente chapa y pintura sino, digamos, de varias cosas. Más que nada de inyección electrónica y... y todo, viste que ahora vienen todos computarizados... Que me guste, no sé... pero que diga estaría bien laburando en tal lugar, qué sé yo... en alguna dependencia municipal... o pública, cosas así. Que no sé si es un trabajo seguro, pero... que te pueden pagar bien... pública y también puedo hacer cualquier trabajo administrativo [...].

En general, priorizan criterios asociados al tipo de tareas, como la comodidad o la menor exposición al esfuerzo físico extremo o la "limpieza" de las mismas (haciendo un contrapunto con la experiencia de la mayoría de los varones en la construcción, y de las mujeres en el servicio doméstico). También aspiran a mejores condiciones laborales, asociadas al imaginario de empleo que muchas veces transmite el PJMyMT: estabilidad, mayor calificación, etc.

Respecto de esto último, en sus relatos la titulación secundaria aparece, a la vez, como un elemento muy valorado en cuanto a la formación necesaria para acceder al a mejora a la que aspiran en cuanto a sus puestos de trabajo. ${ }^{20}$ Pero, por otra parte, pudimos observar una postura reflexiva y crítica respecto de las implicancias últimas de la titulación secundaria: no solamente por su valor formativo, sino fundamentalmente por su valor simbólico y por la certificación de marcas sociales, además de educativas: "ser alguien”, más allá de las necesidades de los saberes escolares para determinados tipos de tareas (como las de reposición o la atención al cliente).

No, decidí volver al colegio porque yo a la semana que dejé el colegio me arrepentí. Pero ya iba muy mal en el colegio y me iba a quedar otro año, así que decidí volver al colegio porque quería seguir estudiando. Además es cierto eso que te dicen que sin el colegio, sin el secundario no sos nada, porque es así, no podes hacer otra $\cos a[\ldots]$.

Finalmente, encontramos cierta recurrencia en cuanto a categorizaciones de "tipos" de trabajos, a partir de componentes que permiten distinguir distintos grados de legitimidad y valoración de las actividades realizadas.

Y...como te decía, el trabajo fácil es el trabajo que te decía yo, y ese trabajo los lleva porque la sociedad, la sociedad lo está, como que no quiere estudiar, los padres como que generan...si el gobierno genera vagancia mas se va a producir más vagancia, y si están podridos los de arriba se van a podrir todos los de abajo".

O sea, no son trabajos buenos que te paguen bien, ¿entendés? Que puedas llegar a avanzar, a avanzar vos como persona o a avanzar en tu trabajo. Porque si una persona trabaja de albañil, va a ser toda la vida albañil; una persona que trabaja limpiando una casa, si no haces algo vos, vas a limpiar toda tu vida una casa, ¿entendés? Pero si trabajas, ponele, empezás, qué sé yo, repartiendo cartas en una oficina y avanzas, ponele, que podés llegar a ser secretaría o podés llegar a algo más. Y vos te ponés expectativas y vos luchas por eso, obvio, porque no todo te va a venir de arriba [...]. No, pero...el trabajo que es como yo sigo, mediocre, que no es la apalabra que quiero usar pero bueno, no me sale otra, son trabajos que no podés avanzar. No avanzás ni vos como persona ni avanzás en tu trabajo. Es así, es lo que yo pienso.

Así, los “trabajos fáciles" aparecen como aquellas actividades ilegales a las que muchos jóvenes se ven forzados, de acuerdo a los entrevistados, debido a circunstancias sociales que los exceden pero que en alguna medida los condenan (deserción escolar que los condena al desempleo, relaciones familiares conflictivas, situación socio-política, etc.). ${ }^{21}$ Mientras tanto,

20. Recordamos que uno de los principales servicios prestados por el PJMyMT y en el que más hace hincapié su diagnóstico es el del apoyo a la terminalidad educativa.

21. Tal como señala en su investigación Kessler (2004), el hecho de que existan categorías diferenciales no quiere decir que las prácticas así clasificadas sean mutuamente excluyentes. El imaginario histórico de ladrones profesionales que ejercen el delito como actividad a tiempo 
los "trabajos mediocres" serían aquellas actividades que demandan escasa calificación y son relativamente sencillos de conseguir pero que no permiten una carrera laboral "ascendente" o un desarrollo personal (en general, aquellas a las que se han visto expuestos y en las que tienen experiencia). Si antes describíamos una serie de actividades valoradas por su carácter y sus condiciones, aquí observamos también la categorización negativa de otros trabajos.

Estas significaciones se encadenan con relatos acerca de elecciones individuales donde se prioriza el dinero fácil sin esfuerzo (delincuencia o "vagancia") o la falta de expectativas y de "lucha" para alcanzar esas metas (trabajos mediocres). Nuevamente la voluntad individual y el mérito ganan un lugar central en las miradas sobre el trabajo:

Yo tengo el pensamiento, como te dije, yo tengo el pensamiento de que si vos querés trabajar vas a trabajar, es así. De lo que sea vas a conseguir un trabajo Yo creo que hay que ir escalando y ponerse una meta, de decir: "Bueno, hoy empiezo acá abajo, pero tarde o temprano quiero llegar allá y...bueno, tengo que estudiar, hacer uno que otro cursito que me ayude, que me dé más puntos, que me dé más experiencia, lo haremos", pero yo creo que todo está en nosotros. Si nosotros decimos: "Bueno, lo hago, es asî". Si nosotros nos ponemos en que: "No, me da fiaca, mejor acá que estamos en negro, más fácil si, te llaman más rápido" o "Están llamando más gente, seguro que entro", no sé...

\section{Condición juvenil: entre lo apropiado y lo propio}

Tal como planteamos anteriormente, la mirada pesimista de la juventud del programa la caracteriza como un grupo poblacional sin visión de futuro, caracterizado por la inmediatez de sus decisiones, fundamentalmente las laborales. Cuando indagamos a los entrevistados acerca de la condición de juventud, las respuestas tendieron a reproducir gran parte de este imaginario sostenido por funcionarios del programa:

Y, porque lamentablemente... lamentablemente la juventud de ahora está arruinada. En Córdoba hay mucha droga. Eso maneja todo. Mucha droga y no hay seguridad. Por ahí hay mucho policía que están incorporados al tema de la droga... [...] sí, hay personas que no quieren trabajar, que quieren estar... en una palabra, estar al cuete, digamos. Estar al día, vivir la vida, que... dar vuelta en su casa, en el barrio, sin hacer nada, digamos. Y sí, hay mucha gente que... bah, los chicos jóvenes, que no quieren trabajar, digamos.

Que se cansan rápido, que buscan el trabajo, a veces, solamente para, para tener plata para algo y para comprarse esto y lo otro y qué sé yo, esas cosas y después se dan cuenta que se cansan y bueno, eso. Tampoco... porque también a veces, muchas veces es difícil porque como antes no tuvieron, la familia no lo influyó en que estudie o busque algún trabajo mejor o que tenga, no sé, otras expectativas, entonces a la hora de buscar trabajo ya no tiene experiencia laboral, ni el colegio muchas veces terminado [...] Los jóvenes de hoy en día no quieren trabajar, viven para la joda. O sea, está muy perdida la juventud hoy en día, es así.

Una juventud significada como perdida, arruinada por la droga, dedicada a la "joda" y sin una familia que la contenga y oriente, da cuenta de una gran efectividad del programa como modo de generación, es decir, de enclasamiento de sujetos según su edad, que les atribuye una serie de características negativas. Sin embargo, los relatos muestran, cuando no se refieren a "otros" jóvenes, sino a las propias trayectorias, elementos de planificación y proyección que disienten del diagnóstico propuesto por el PJMyMT respecto de la preeminente inmediatez como la temporalidad que define a la juventud, aun cuando dichas proyecciones se construyan en ámbitos de inestabilidad o precariedad. Las rupturas que los jóvenes sostienen en sus discursos respecto a los puestos y las actividades laborales disponibles se refieren a la estabilidad o la rutina como norma social. Muchos jóvenes manifiestan preferencia por tareas que no impliquen monotonía o "encierro" y apuestan a una idea de "autogestión", "autonomía", que entran en consonancia con la promoción del "autoempleo" y la edificación de "empresarios de sí mismos", propia del discurso del PJMyMT.

Trabajaría con esas cosas, pero no en algo monótono, si no en algo variado [...]. Soy inquieta [...] y después quería también hacer, estaba con dudas porque también quería hacer, yo quería hacer un propio negocio mío con ayuda de mi mamá.

No sé, el hecho de trabajar afuera ya, por ejemplo, de estar al aire libre, ya, así esté parada y tenga que frenar a alguien y pedirle los papeles [...]. Igual que la caminera, decir, estoy al aire libre, no estoy encerrada entre cuatro paredes al frente de una computadora, o atendiendo un teléfono, que tenés que aguantarte a la

completo ocluyó la posibilidad de captar oscilaciones o "movilidades laterales" (Kessler, 2013) de sujetos que combinaban actividades laborales legales e ilegales, escuela y delito, trabajo y delito, etc., y suponiendo que el mundo del trabajo era una realidad ajena y paralela a quienes ejercían la delincuencia. 
viejitas que te molestan, o trabajar en un call [...] debe estar bueno el hecho de trabajar al aire libre y tener, qué sé yo...para mí ya el aire es un privilegio."

\section{Conclusión}

Incorporar las voces de los beneficiarios en una lectura del funcionamiento de este programa de empleo implica reintroducir la complejidad de las derivas, contradicciones y contingencias que constituyen la configuración del dispositivo en un conjunto de prácticas que generan encuentros y desencuentros con la direccionalidad de acciones propuestas por esta política.

Si bien esta mirada sobre la política repara sobre los momentos de transición y las estrategias de inserción de los jóvenes, pone en el centro de su atención los modos de producción de esos sujetos como "jóvenesen-transición”, deteniendo su mirada en una serie de agentes, instituciones y mecanismos que participan en este proceso, entre los cuales incluimos a los mismos beneficiarios.

A partir de esto entendemos que entre el campo de acción pretendido, producido y regulado por el PJMyMT y los sentidos atribuidos a las prácticas laborales entre los jóvenes del programa, encontramos tensiones que definen los pesos, los límites y las posibilidades de la política para producir criterios de validez y legitimidad en torno al trabajo.

Una primera tensión la encontramos en torno al enfoque de la empleabilidad: mientras que el programa centra su foco de intervención en los hábitos, las actitudes, competencias y conocimientos certificados, se marginaliza, de cierta forma, la posibilidad de transformar y trabajar sobre las prácticas de inserción y búsqueda laboral efectivas de los jóvenes. Esto produce una serie de dificultades al momento de vincular e intervenir la formación y capacitación certificada, las "nuevas" competencias en búsqueda y presentación formal de trabajo, con prácticas efectivas de inserción fundamentalmente habilitadas por contactos y redes personales y familiares, signadas por la informalidad (tanto en las condiciones laborales como en la tipología de vínculo establecido). En este sentido, creemos necesario profundizar evaluaciones sobre las posibilidades efectivas que el dispositivo tiene de modificar las trayectorias laborales de los jóvenes, atendiendo tanto a la reproducción de los sentidos y lógicas instaladas en sus trayectorias familiares previas como a las condiciones estructurales de un mercado de trabajo que continúa siendo segmentado y heterogéneo.

La segunda de las tensiones se sitúa a nivel de la producción de la población beneficiaria. Como mencionamos a lo largo de este trabajo, la lógica político-institucional propia del Estado produce y reproduce una construcción ideológica hegemónica en torno a la juventud vulnerable, que los conceptualiza (en relación al trabajo) en base a una serie de carencias o faltas: de hábitos, valores, competencias básicas y capacidades certificadas, anclado en el imaginario de segmento formal y protegido del mercado de trabajo. A partir de esto, el programa y su diagnóstico sobre la problemática de empleo juvenil, define y produce como no-trabajo toda una serie de prácticas y lógicas que, en las trayectorias de los jóvenes, están vinculadas no tanto a carencias o ausencia de anclaje en el mundo del trabajo, sino a las condiciones laborales y al sector informal y precario en el que se insertan sus familias.

Para finalizar, entendemos que estas dos tensiones definen una de las aristas de los modos de generación, específicamente aquella vinculada al empleo, dando cuenta y alumbrando formas alternativas de construcción y apropiación de la condición juvenil en torno a sus prácticas en el mundo del trabajo, en relación a la familia, etc. En otras palabras, consideramos que esta lectura sobre los dispositivos no sólo pretende desnaturalizar aquellas construcciones hegemónicas que operan en los procesos de inserción, sino que también permite visibilizar, a partir de las fugas, esas formasotras de ser joven y de trabajar y significar el trabajo.

\section{Referencias}

ASSUSA, G.; BRANDÁN ZEHNDER, M. G. Salvar a la generación perdida. Gubernamentalidad, empleabilidad y cultura del trabajo en un programa de empleo para jóvenes de Argentina. Sociología e Política, Universidade Federal do Paraná, Brasil (en prensa), 2014.

AVALLE, G.; BRANDÁN ZEHNDER, M. G. Entre la compensación y la inclusión. Tensiones en las políticas laborales y de empleo en la Argentina post-convertibilidad. Perspectivas de Políticas Públicas, n. 1, año 1. Julio-Diciembre, Buenos Aires, p. 29-46, 2011.
BENDIT, R.; STOKES, D. Jóvenes en situación de desventaja social: políticas de transición entre la construcción social y las necesidades de una juventud vulnerable. Estudios de Juventud, n. 65, Instituto de la Juventud, España, 2004.

BURGOS, A.; ROBERTI, E. Los mundos del trabajo: multiplicidad de dispositivos, trayectorias y sentidos en la inserción laboral de los jóvenes. Ponencia presentada en el $11^{\circ}$ Congreso Nacional de Estudios del Trabajo. 2013. ASET. Disponible en: http://www.aset.org.ar/2013/ 
ponencias/p7_Burgos.pdf.

CASAL, J. et al. Aportaciones teóricas y metodológicas a la sociología de la juventud desde la perspectiva de la transición. Papers de Sociología, Universidad Autónoma de Barcelona, n. 79, 2006.

CHÁVES, M. Jóvenes, territorios y complicidades: una antropología de la juventud urbana. Buenos Aires: Espacio Editorial, 2010.

CORRIGAN, P.; SAYER, D. El gran arco. La formación del Estado inglés como revolución cultural. In: LAGOS, M. L.; CALLAS, P. (Comp.). Cuadernos del futuro n. 23. Antropología del Estado. Dominación y prácticas contestatarias en América Latina. La Paz: INUH/PNUD, 2007.

DÁVILA, O.; GHIARDO, F.; MEDRANO, C. Los desheredados: trayectorias de vida y nuevas condiciones juveniles. Valparaíso: Ediciones CIDPA, 2006.

FOUCAULT, M. El sujeto y el poder. In: DREYFUS, H. L.; RABINOW, P. (Orgs.). Michel Foucault: beyond structuralism and hermeneutics (Michel Foucault: más allá del estructuralismo y la hermeneútica). Chicago: Chicago University Press, 1983.

Nacimiento de la biopolítica. Buenos Aires: Fondo de Cultura Económica, 2004.

Seguridad, territorio y población: curso en el Collège de France 1977-1978. Buenos Aires: Fondo de Cultura Económica, 2009.

Defender la sociedad. Buenos Aires: Fondo de Cultura Económica, 2010.

El poder, una bestia magnifica: sobre el poder, la prisión y la vida. Buenos Aires: Siglo XXI, 2012.

JACINTO, C. Los dispositivos recientes de empleo juvenil: institucionalidades, articulaciones con la educación formal y socialización laboral. Revista del Trabajo: Nueva Época, n. 6, año 4, Buenos Aires: MTESS, 2008.

Veinte años de formación para el empleo de jóvenes vulnerables en América Latina: persistencias y reformulaciones. In: (Comp.). La construcción social de las trayectorias laborales de jóvenes: políticas, instituciones, dispositivos y subjetividades. Buenos Aires: Teseo - IDES, 2010.

JACINTO, C.; MILLENAAR, V. Desigualdad social $y$ género en las trayectorias laborales de jóvenes de sectores populares: el lugar de los dispositivos de inserción. Ponencia presentada en el $11^{\circ}$ Congreso Nacional de Estudios del Trabajo. ASET. 2013. Disponible en: <http://www.aset. org.ar/2013/ponencias/p7_Millenaar.pdf>.

JOSEPH, G. M.; NUGENT D. Cultura popular y formación del Estado en el México revolucionario. In:

(Comps.). Aspectos cotidianos de la formación del estado: la revolución y la negociación del mando en el México moderno. México DF: Ediciones Era, 2002.

KESSLER, G. Sociología del delito amateur. Buenos Aires: Paidós, 2004.

Ilegalismos en tres tiempos. In: CASTEL, R.; KESSLER, G.; MERKLEN, D.; MURARD, N. Individuación, precariedad, inseguridad ¿Desinstitucionalización del presente? Buenos Aires: Paidós, 2013.

LLEVADOT, L. El estatuto de ficción en Nietzsche y Foucault. Convivium: Revista de Filosofía, n. 21, p. 71-82, 2008. MADOERY, O. Informe Programa MTEySS-OIT. Más y mejor trabajo para todos. Del Programa Jefes de Hogar al Programa Jóvenes. Políticas activas de empleo, Argentina 2003-2010. 1. ed. Buenos Aires: Programa Cea-OIT, 2011. MARTÍN CRIADO, E. Producir la juventud: crítica de la sociología de la juventud. Madrid: Ed. Itsmo, 1998.

El paro juvenil no es el problema, la formación no es la solución. In: CACHÓN, L. (Coord.). Juventudes, mercados de trabajo y políticas de empleo. Valencia: 7imig. 1999. PÉREZ, P. Los sospechosos de siempre: los desocupados de larga duración en Argentina y su (in)empleabilidad. In: NEFFA, J. C. et al. (Orgs.). Desequilibrios en el mercado de trabajo argentino: los desafíos en la postconvertibilidad. Buenos Aires: CEIL-PIETTE/CONICET, 2005.

RODRÍGUEZ, E. Empleo y juventud. Muchas iniciativas y pocos avances. Nueva Sociedad, n. 232, 2011.

ROSEBERRY, W. Hegemonía y lenguaje contencioso. In: JOSEPH, G. M.; NUGENT, D. (Comps.). Aspectos cotidianos de la formación del estado: la revolución y la negociación del mando en el México moderno. México DF: Ediciones Era, 2002.

SALVIA, A. Jóvenes promesa: trabajo, educación y exclusión social en jóvenes pobres en la Argentina. Buenos Aires: Ed. Miño y Dávila, 2008.

. Juventudes, problemas de empleo y riesgos de exclusión social: el escenario de crisis mundial en la Argentina. Estudio publicado por Friedrich Ebert Stiftung. 2013. Disponible em: <http://library.fes.de/pdf-files/iez/09698. pdf>.

SHORE, C. La antropología y el estudio de la política pública: reflexiones sobre la "formulación" de las políticas. Antípoda: Revista de Antropología y Arqueología, n. 10, enerojunio, p. 21-49, Bogotá: Universidad de Los Andes, 2010. TASSIN, E. De la subjetivación política. Althusser/ Rancière/Foucault/Arendt/Deleuze. Revista de Estudios Sociales, n. 43, Universidad de Los Andes, 2012.

\section{Fuentes}

Ministerio de Trabajo, Empleo y Seguridad Social de la Nación. Disponível em: <http://www.trabajo.gov.ar/>.

Programa AREA - OIT: Aportes a las políticas activas de empleo en la Argentina. Informe final del Programa AREA. 1. ed. Buenos Aires, 2008.

OIT. Trabajo decente para los jóvenes: el desafío de las políticas de mercado de trabajo en Argentina. Notas OIT: trabajo decente en Argentina. Buenos Aires, 2011.

OIT. Informe la crisis del empleo de los jóvenes: ¡Actuemos ya! Informe $V$ de la Conferencia Internacional del Trabajo, 101 ${ }^{\mathrm{a}}$ Reunión. 2012.

OIT. Informe tendencias mundiales del empleo juvenil 2013: una generación en peligro. 2013a.

OIT. Notas sobre trabajo decente para los jóvenes: el desafío de las políticas de mercado de trabajo en Argentina. 2013b. 
Employability, meanings and devices: an analysis from the perspective of the beneficiaries from the Programa Jóvenes Más y Mejor Trabajo in

\title{
Córdoba Capital, Argentina
}

\begin{abstract}
In the last decade we have witnessed a reconfiguration of the employment policies and the place of the State as regulator of labor practices. Under the paradigm of active employment policies, public intervention on the youth employment problems has been made under the category of employability, focusing on orientation and induction to the workplace process. From a perspective that thinks employment public policies as subjectivation devices, this text retrieves analyzes on a youth employment program of Argentina, to focus on the point of view from the beneficiaries and analyze their meanings production about work, within the frame enabled by the program.
\end{abstract}

Key words: youth employment, youth policies, employability, devices, generation modes.

Empregabilidade, sentidos e dispositivos: um estudo de caso a partir da perspectiva dos beneficiários do Programa Jóvenes Más y Mejor Trabajo em Córdoba Capital, Argentina

\section{Resumo}

A última década assistiu a uma reconfiguração das políticas de emprego e do Estado como regulador das práticas do trabalho. Sob esse paradigma, a intervenção no emprego dos jovens tem ocorrido com foco no processo de orientação e indução ao mundo do trabalho. A partir de uma perspectiva de políticas públicas de emprego, entendidas como dispositivos de subjetivação, este texto recupera a análise de programa de emprego para os jovens da Argentina, para focar o olhar dos beneficiários e analisar o movimento e produção de sentidos sobre o trabalho habilitado dentro do programa.

Palavras-chave: emprego da juventude, políticas públicas de juventude, dispositivos, empregabilidade, modos de geração. 